\title{
Aplicação da Função de Lyapunov num Problema de Controle Ótimo de Pragas
}

C. CODEN FELTRIN ${ }^{1}$, M. RAFIKOV ${ }^{2}$, Departamento de Física, Estatística, e Matemática, UNIJUÍ, 98700-000 Ijuí, RS, Brasil.

Resumo. Para reduzir a população de pragas na lavoura e substituir agrotóxicos químicos, cada vez mais o agricultor busca o controle biológico. Usualmente, para realizar o controle biológico, parasitóides e predadores são criados em laboratórios. Ao atingir um número adequado de inimigos naturais eles são liberados com a expectativa que um sistema presa-predador ou hospedeiro-parasitóide seja criado.

Em [6] mostra-se que funções de Lyapunov podem ser usadas para investigar a estabilidade de vários modelos presa-predador. Neste trabalho foi considerado um problema de otimização de controle que utiliza, por um lado uma função de Lyapunov e, por outro lado as idéias de otimização de Bellman [1]. Neste problema, foi encontrada uma função de controle ótimo que determina a quantidade de inimigos naturais de pragas que devem ser liberados na lavoura, mantendo a população de pragas abaixo do nível de danos econômicos.

\section{Introdução}

Dentro do controle biológico existem três tipos a considerar [3][4]:

i) Controle biológico clássico envolve a investigação a respeito da origem da praga, a fim de descobrir quais são seus inimigos naturais. Indivíduos da espécie inimiga ( normalmente exótica ) são trazidos (importados) para que naturalmente se reproduzam e ataquem a praga. Este é o tipo de controle biológico mais antigo e que tem tido mais sucesso recentemente.

ii) Aumento é um método mais recente, que consiste na liberação de grandes quantidades de inimigos naturais, os quais são produzidos em massa em laboratórios. A liberação pode ser feita apenas no período e local em que for necessária . Este método exige uma contínua manutenção e não fornece uma solução permanente.

iii) Controle de conservação enfatiza a manipulação de aspéctos de ecossistema para conservar e aumentar populações de inimigos naturais de modo que os problemas com a praga fiquem reduzidos.

No controle clássico e de aumento geralmente são liberadas grandes quantidades de inimigos naturais na lavoura, na intensão de que nenhuma praga escape ao

\footnotetext{
${ }^{1}$ feltrin@detec.unijui.tche.br

${ }^{2}$ rafikov@main.unijui.tche.br
} 
ataque. Em muitos casos, esta técnica leva a um desequilíbrio, sendo que ambas as espécies tendem a ser extintas. Neste caso, as pragas podem recolonizar a lavoura, não havendo então inimigos naturais para combatê-las. Uma visão ecológica considera um inseto como praga se e somente se a quantidade deste inseto na lavoura causa danos econômicos. O objetivo deste trabalho é encontrar uma estratégia do controle ótimo de pragas através da liberação de inimigos naturais que leve a um estado de equilíbrio positivo em que a densidade de pragas se estabilize sem causar danos econômicos, e que a população de inimigos naturais se estabilize em um valor suficiente para controlar as pragas.

\section{Modelos Matemáticos}

As interações entre pragas e seus inimigos naturais numa lavoura são representadas por um modelo do tipo presa-predador.

$$
\begin{aligned}
& \frac{d x}{d t}=x f_{1}(x, y), \\
& \frac{d y}{d t}=y f_{2}(x, y) .
\end{aligned}
$$

Neste trabalho é considerado um caso particular do modelo (2.1):

$$
\begin{aligned}
& \frac{d x}{d t}=x(a-\gamma x-\alpha y), \\
& \frac{d y}{d t}=y(-b+\beta x),
\end{aligned}
$$

onde $a, \gamma, \alpha, b$ e $\beta$ são constantes positivas com valores conhecidos.

O sistema (2.2) caracteriza interações entre pragas e seus inimigos naturais sem controle.

As variáveis x e y representam as densidades das presas e predadores; os produtos ax e -by modelam a taxa de crescimento das presas e a taxa de mortalidade dos predadores, respectivamente; os termos - $\alpha x y$ e $\beta x y$ representam as interações entre as duas populações em ambas as equações, onde cada encontro tende a ser favorável ao predador pois possibilita o crescimento dessa espécie e desfavorável à presa por reduzir a sua população; o produto $(-\gamma x) x$ determina a competição intraespecífica.

Harrison [7] mosrou que a condição $\gamma>0$ é suficiente para a estabilidade global do sistema (2.2). Mesmo sendo sempre estável, o modelo (2.2) pode possuir o ponto de equilíbrio do sistema a níveis muito altos. Neste caso há necessidade de aplicar controle.

Existe um certo limite de quantidade de pragas que caracteriza a margem de danos econômicos que vamos designar $x_{d}$. Por exemplo, conforme informações da EMBRAPA, a densidade de 20 lagartas de soja grandes por metro quadrado (com mais de $1,5 \mathrm{~cm}$ ) ou a densidade de 40 lagartas pequenas ( de 0,5 a $1,5 \mathrm{~cm}$ ) por metro 
quadrado não causam danos econômicos a lavoura, ou seja, neste caso $x_{d}=20 \mathrm{ou}$ $x_{d}=40$ para lagartas de soja grandes e pequenas, respectivamente.

Para manter o sistema num ponto de equilíbrio abaixo de danos econômicos temos que introduzir uma função de controle no sistema (2.2) que caracteriza o número de inimigos naturais liberados no sistema no instante t.

O modelo que descreve a dinâmica do sistema com aplicação de controle pode ser escrito da seguinte forma:

$$
\begin{aligned}
& \frac{d x}{d t}=x(a-\gamma x-\alpha y), \\
& \frac{d y}{d t}=y(-b+\beta x+U) .
\end{aligned}
$$

A função de controle $U$ consiste de duas partes e tem a seguinte forma

$$
U=\delta y+u,
$$

onde a parte $\delta y$ representa a introdução de inimigos naturais para levar o sistema ao ponto de equilíbrio desejado, e a parte $u$ garante a estabilidade deste ponto de equilíbrio.

\section{Controle Ótimo de Pragas e Análise de Estabi- lidade Através da Função de Lyapunov}

O ponto de equilíbrio desejado $P=\left(x^{*}, y^{*}\right)$ pode ser encontrado da primeira equação do sistema (2.3), considerando $x^{*}=x_{d}$ e $\frac{d x}{d t}=0$, ou seja:

$$
a-\gamma x_{d}-\alpha y^{*}=0 .
$$

De (3.1) resulta:

$$
y^{*}=\frac{a-\gamma x_{d}}{\alpha}>0 .
$$

Geralmente, o coeficiente de competição entre as pragas $\gamma$ é bastante pequeno, e a condição de positividade

$$
a-\gamma x_{d}>0
$$

é satisfeita. Considerando que $u \rightarrow 0$ quando $t \rightarrow \infty$, da segunda equação do sistema (2.3) obtém-se:

$$
-b+\beta x^{*}+\delta y^{*}=0
$$

de onde resulta:

$$
\delta=\frac{b-\beta x_{d}}{y^{*}} .
$$


Como é visto de (3.5) o coeficiente $\delta$ tem que ser positivo, pois caso contrário será

$$
x_{d}>\frac{b}{\beta},
$$

onde $\frac{b}{\beta}$ é o nível de equilíbrio de pragas sem controle. Então, a desigualdade (3.6) significa que o nível de equilíbrio de pragas desejado é maior que o nível de equilíbrio de pragas sem controle e o problema neste caso perde o sentido.

Objetivando agora encontrar $u$ foi formulado o seguinte problema de controle ótimo:

Encontrar a função de controle $u$ que minimiza o funcional

$$
I=\int_{0}^{\infty}\left[m_{1}\left(x-x^{*}\right)^{2}+m_{2}\left(y-y^{*}\right)^{2}+u^{2}\right] d t
$$

e estabiliza o sistema (2.3) no ponto de equilíbrio $P=\left(x^{*}, y^{*}\right)$ abaixo de danos econômicos.

No funcional (3.7), $m_{1}$ e $m_{2}$ são constantes positivas. Para resolver este problema foi utilizada a Programação Dinâmica [1], interpretada em termos da teoria de controle ótimo [2].

Conforme este método, o problema formulado do controle ótimo reduz-se à resolução da seguinte equação diferencial parcial de Hamilton-Jacobi-Bellman[1].

$$
\min _{u}\left(\frac{d V}{d t}+w\right)=0
$$

onde

$$
w=m_{1}\left(x-x^{*}\right)^{2}+m_{2}\left(y-y^{*}\right)^{2}+u^{2} .
$$

A função $V$ por definição é:

$$
V(x(t))=\min _{u} \int_{t}^{\infty}\left[m_{1}\left(x-x^{*}\right)^{2}+m_{2}\left(y-y^{*}\right)^{2}+u^{2}\right] d t .
$$

É óbvio que a relação entre $V$ e o valor ótimo do critério de otimização (3.7) é a seguinte:

$$
I\left[u_{0}\right]=V(x(0)) .
$$

Num caso geral a equação de Hamilton-Jacobi-Bellman(3.8) é uma equação diferencial parcial e ainda com operador de minimização. Sabe-se que a solução $V(x, t)$ da equação $(3.8)$ de um problema de controle ótimo para o sistema linear com o funcional quadrático pode ser procurada como uma forma quadrática que, por um lado, satisfaz a equação (3.8) e, por outro lado, é uma função de Lyapunov[8]. No trabalho [9] foi proposto procurar a solução da equação (3.8) entre funções de 
Lyapunov para modelos do tipo Lotka-Volterra. Para o caso não linear do sistema (2.3) a solução $V(x, t)$ da equação (3.8) foi procurada na seguinte forma:

$$
V(x, y)=v_{1}\left(x-x^{*}-x^{*} \ln \left(\frac{x}{x^{*}}\right)\right)+v_{2}\left(y-y^{*}-y^{*} \ln \left(\frac{y}{y^{*}}\right)\right),
$$

onde $v_{1}$ e $v_{2}$ são constantes positivas a determinar.

A função de Lyapunov do tipo (3.12) fo utilizada nos trabalhos [6] e [11] para demonstrar a estabilidade global do sistema de Lotka -Volterra. O objetivo deste trabalho é encontrar os valores $v_{1}$ e $v_{2}$ que satisfazem a equação de Hamilton Jacobi-Bellman.

Considerando a derivada de (3.12) ao longo das trajetórias do sistema (2.3), resulta:

$$
\frac{d V}{d t}=v_{1}\left(x-x^{*}\right)(a-\gamma x-\alpha y)+v_{2}\left(y-y^{*}\right)(-b+\beta x+\delta y+u) .
$$

Substituindo os resultados para $w(3.9)$ e $\frac{d v}{d t}(3.13)$ na equação de HamiltonJacobi-Bellman(3.8), obtém-se:

$$
\begin{array}{r}
\min \left[v_{1}\left(x-x^{*}\right)(a-\gamma x-\alpha y)+v_{2}\left(y-y^{*}\right)(-b+\beta x+\delta y+u)\right. \\
\left.+m_{1}\left(x-x^{*}\right)^{2}+m_{2}\left(y-y^{*}\right)^{2}+u^{2}\right]=0 .
\end{array}
$$

A função $u(t)$ não é limitada e pode ser encontrada da equação:

$$
\begin{array}{r}
\frac{\partial}{\partial u}\left[v_{1}\left(x-x^{*}\right)(a-\gamma x-\alpha y)+v_{2}\left(y-y^{*}\right)(-b+\beta x+\delta y+u)\right. \\
\left.+m_{1}\left(x-x^{*}\right)^{2}+m_{2}\left(y-y^{*}\right)^{2}+u^{2}\right]=0,
\end{array}
$$

de onde segue

$$
u_{0}=\frac{-v_{2}}{2}\left(y-y^{*}\right),
$$

onde $u_{0}$ é o valor ótimo da função de controle; o valor de $u_{0} y$ representa o fluxo de inimigos naturais que devem ser introduzidos no sistema em cada instante $t$.

Substituindo $u=u_{0}$ na equação (3.8), obtém-se:

$$
\frac{d V}{d t}+w=0
$$

ou

$$
\begin{aligned}
& v_{1} \gamma\left(x-x^{*}\right)^{2}-\alpha v_{1}\left(x-x^{*}\right)\left(y-y^{*}\right)+\beta v_{2}\left(y-y^{*}\right)\left(x-x^{*}\right) \\
& \quad+\left(\delta v_{2}-\frac{v_{2}^{2}}{2}\right)\left(y-y^{*}\right)^{2}+m_{1}\left(x-x^{*}\right)^{2}+m_{2}\left(y-y^{*}\right)^{2}+\frac{v_{2}^{2}}{4}\left(y-y^{*}\right)^{2}=0 .
\end{aligned}
$$


Por comparação, resulta

$$
\begin{gathered}
-\gamma v_{1}+m_{1}=0, \\
v_{2}^{2}-4 \delta v_{2}-4 m_{2}=0, \\
-v_{1} \alpha+v_{2} \beta=0 .
\end{gathered}
$$

As raízes da equação (3.20) são

$$
v_{2}=2\left(\delta \pm \sqrt{\delta^{2}+m_{2}}\right) .
$$

Para que $v_{2}>0$, escolhe-se

$$
v_{2}=2\left(\delta+\sqrt{\delta^{2}+m_{2}}\right) .
$$

Da equação (3.21) obtém-se

$$
v_{1}=\frac{\beta}{\alpha} v_{2}
$$

e, da equação (3.19), obtém-se

$$
m_{1}=\gamma v_{1} .
$$

Finalmente, analisa-se a estabilidade do sistema (2.3) através da função de Lyapunov. A função $V(x, t)$ é definida positiva por definição. O sistema (2.3) com controle ótimo (3.16) será assintoticamente estável se $\frac{d V}{d t}$ for definida negativa.

Substituindo $v_{1}, v_{2}$ e $u$ em $\frac{d V}{d t}$, obtém-se:

$$
\frac{d V}{d t}=-m_{1}\left(x-x^{*}\right)^{2}-\left(\sqrt{\delta^{2}+m_{2}}\right)\left(y-y^{*}\right)^{2}<0 .
$$

Como $m_{1}, m_{2}$ e $\delta$ são constantes positivas, pode-se afirmar que $\frac{d V}{d t}$ é definida negativa.

Os cálculos podem ser efetuados na seguinte ordem:

1. Escolher o coeficiente $m_{2}$;

2. Calcula-se o valor de $v_{2}$ através da equação (3.23);

3. Da equação (3.24) calcula-se $v_{1}$.

4. Calcula-se o valor de $m_{1}$ através da equação (3.25);

5. O controle ótimo para o problema formulado pode ser encontrado da fórmula (3.16). 


\section{Simulações}

Para ilustrar os algoritmos propostos para o problema do controle ótimo acima considerado foram feitas simulações numéricas com parâmetros que estão presentes na Tabela 1.

Tabela 1

\begin{tabular}{|c|c|c|c|c|c|c|}
\hline$y_{0}$ & $a$ & $b$ & $\alpha$ & $\gamma$ & $\beta$ & $x_{d}$ \\
\hline 9,2 & 0,16 & 0,19 & 0,02 & 0,001 & 0,0029 & 20 \\
\hline
\end{tabular}

Os dados apresentados na Tabela 1 correspondem a um sistema com as relações presa-predador entre a lagarta da soja ( Anticarsia gematalis) e seus predadores.

Na Figura 1, estão presentes os resultados de simulações com base no modelo (2.2), sem aplicação de controle biológico. Como é visto dos gráficos da Figura 1 a população de pragas apresenta estabilização num nível $x^{*}=65.51723$. Mas este valor é muito maior que o valor $x_{d}=20$ presente na Tabela 1 , o qual é recomendado pela EMBRAPA.

Para garantir o nível recomendado para o valor desejado de equilíbrio foi escolhido $x_{d}=20$. Este nível de equilíbrio de pragas pode ser controlado por predadores em quantidade $y^{*}=7$, calculado através da fórmula (3.2). Neste caso $\delta$ foi calculado através da fórmula (3.5), onde obteve-se o valor $\delta=0.001886$.

Com este tipo de controle o sistema tem a seguinte forma:

$$
\begin{aligned}
\frac{d x}{d t} & =x(-0.16-0.001 x-0.02 y), \\
\frac{d y}{d t} & =y(-0.19-0.0029 x+0.01886 y) .
\end{aligned}
$$

Para esses parâmetros o modelo apresentou um comportamento instável.

A introdução da função de controle $u$ conforme o algoritmo de otimização acima referido garantiu a estabilidade, como é visto da Figura 2. Na Figura 3, está presente a variação da função de controle ao longo do tempo.

As simulações foram feitas para o valor para o valor $m_{2}=0.00302$. Os valores de parâmetros encontrados através do algoritmo acima referido estão na Tabela 2.

Tabela 2

\begin{tabular}{|c|c|c|}
\hline$m_{1}$ & $v_{1}$ & $v_{2}$ \\
\hline 0,00002 & 0,02232 & 0,15391 \\
\hline
\end{tabular}

A função de controle ótimo neste caso é $u_{0}=-\frac{0.15391}{2}(y-7)$

A realização prática do controle acima referido é possível. A tecnologia de liberação de inimigos naturais pode ser facilmente adaptada para realizar o controle biológico conforme os algoritmos acima referidos. 

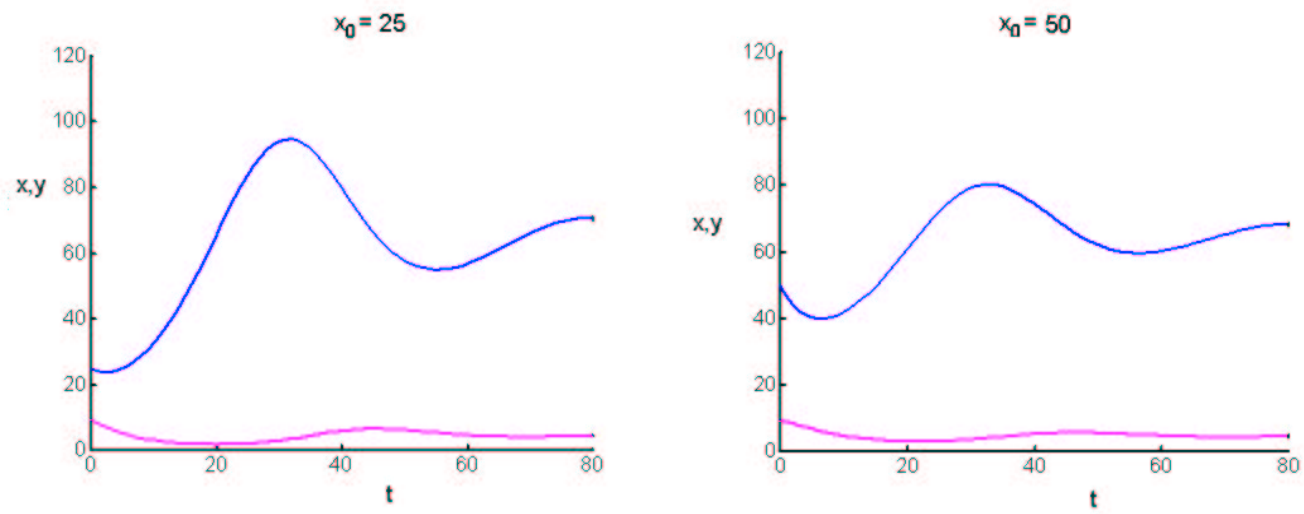

Figura 1: Os inimigos naturais estão representados pela linha tracejada, enquanto que as pragas estão representadas pela linha contínua
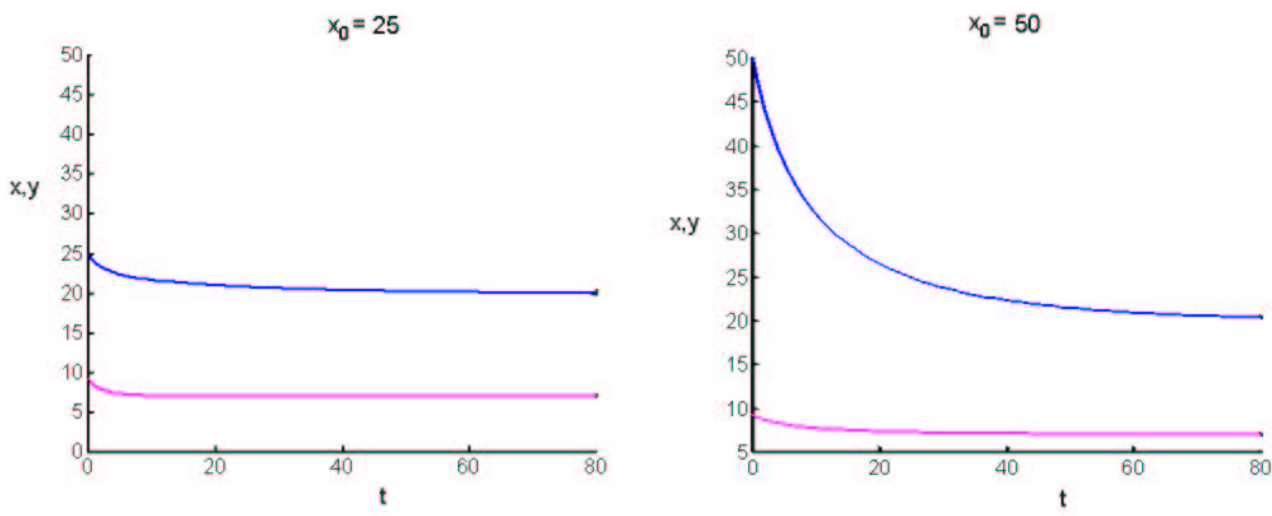

Figura 2: Os inimigos naturais estão representados pela linha tracejada, enquanto que as pragas estão representadas pela linha contínua 

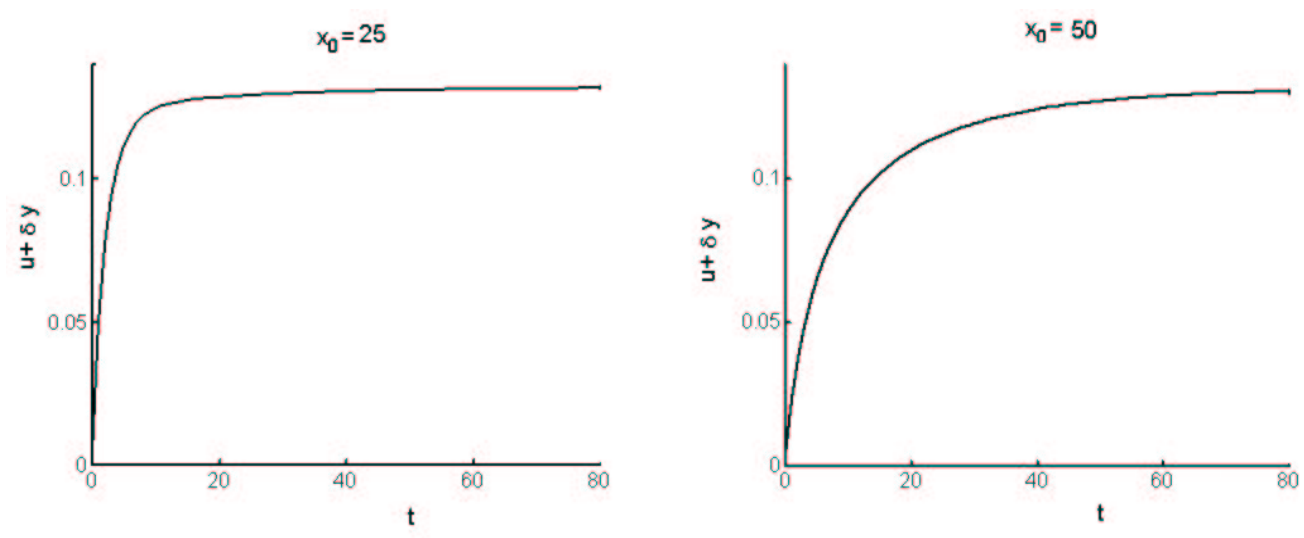

Figura 3:

Como foi mencionado, a idéia de procurar a solução da equação de HamiltonJacobi-Bellman entre as funções de Lyapunov inicialmente foi utilizada para sistemas lineares com o funcional quadrático. Nos últimos anos surgiu o interesse em aplicar essas idéias para sistemas não lineares com o funcional quadrático. Neste trabalho foi apresentada uma abordagem para os sistemas populacionais. Aplicações de funções de Lyapunov ao controle ótimo de outros tipos de sistemas pode ser encontradas nas referências [5] e [10].

Agradecimentos. Os autores agradecem os apoios financeiros recebidos do CNPq e FAPERGS durante a realização deste trabalho.

Abstract The farmer has increasingly been using biological control to reduce the pest population in crop, and to substitute the agrochemicals. Usually, the bilogical control is performed with predators and parasitoidas developed in laboratories. When an adequated amount of them is ready, they are released, and the farmer expects the creation of a prey-predator or a host-parasitoid system.

It is shown in [6] that the use of Liapunov functions to investigate the stability of several prey-predator models. This work considers a control optimization problem, witch uses both a Liapunov function and the Belmann optimization approach [1]. It was found an optimal control function to this problem, witch determines the amount of natural enemies that should be released in the crop, keeping the pest population below the economic damage level.

\section{Referências}

[1] R. Bellman, "Dynamic Programming", Princeton, New Jersey, 1957.

[2] A.E. Bryson and Y.C. Ho, "Applied Optimal Control", Blaisdell, Waltham. Mass., 1969. 
[3] P. DeBach, The scope of biological control, in: "Biological Control of Insects Pests and Weeds" (P. DeBach Ed.), pp.3-20, Chapman \& Hall, London, U.K., 1964.

[4] D. Dent, Ed., Integrated Pest Management, pp. 356, Chapman and Hall, London, U.K.

[5] R. Freeman and J, Primbs, Control Lyapunov functions: New ideas from and old sourse, in "Proceedings of the 35 IEEE Conference on Decision and Control", pp. 3926-3931, Kobe, Japan, 1996.

[6] B.S. Goh, "Management and Analisys of Biological Population", Elsevier Scientific Publishing company, Amsterdam, 1980.

[7] G.W. Harrison, Global Stability of predator-prey interactions, J. Math. Biology, 8 (1979), 159-171.

[8] A.M. Letov, The analytical design of control systems, Automation and Remote Control, 22 (1961), 363-372.

[9] M. Rafikov e C.C. Feltrin, Otimização do controle biológico de pragas: método de funções de Lyapunov, em "Proceedings do Congresso Latino Americano de Biomatemática, X ALAB-V ELAEM", pp. 71-80, UNICAMP, Campinas, 2001.

[10] E.D. Sontag, A universal construction of Artstein's theorem on nonlinear stabilization, Syst. Contr. Lett., 13, No. 2 (1989), 117-123.

[11] V. Volterra, "Leçons sur la Théorie Mathematique de la Lutte Pour la Vie", Gauthier-Villars, Paris, 1931. 